\title{
EXCHANGE COUPLING OF MAGNETIC MULTILAYERS IN SPIN-POLARIZED JELLIUM
}

\author{
W. SCHMIDT \\ Institute of Molecular Physics, Polish Academy of Sciences \\ Smoluchowskiego 17, 60-179 Poznań, Poland
}

The bilinear and biquadratic coupling between two metallic layers separated by a nonmagnetic metal spacer are studied. The paper contains analytical formulae for the coupling which are obtained from the energy potential of the spin-polarized electron gas. The formulae for the coupling are obtained in the framework of the Hohenberg-Kohn formalism of the density functional theory and variables of the coupling are the electron density and the spin polarization of the magnetic multilayer.

PACS numbers: 75.70.-i, 73.20.-r

In the field of the interlayer coupling of magnetic multilayers, the authors of early papers have assumed that the interlayer coupling energy is Heisenberg-like. However, experiments [1] imply that the Heisenberg-like interlayer coupling is too crude to describe the coupling. Slonczewski [2] proposed that the magnetostatic interaction induced by a roughness can lead to a biquadratic term. Several authors proposed other intrinsic mechanisms (e.g. [3,4]). In this paper we propose analytical formulae for the coupling derived from the energy potential of the spin-polarized electron gas. Spin polarized systems were reviewed by Kohn and Vashishta [5]. We take the spin polarization in the kinetic and exchange terms of the energy potential into account.

In this paper, we consider two ferromagnetic slabs separated by a noble metal layer. Numerical calculations are performed for a trilayer $\mathrm{Fe} / \mathrm{Cu} / \mathrm{Fe}$. The exchange coupling is defined as: $J=-J_{0} \cos \vartheta-J_{1} \sin ^{2} \vartheta$, where $\vartheta$ is an angle between the magnetic moments (polarization directions of the $T_{2 \mathrm{~g}}$ up $d$-electrons). The bilinear, $J_{0}$, and the biquadratic, $J_{1}$, exchange terms are obtained using the energy potential of the spin-polarized electron gas. The spin polarization, $\boldsymbol{m}$, is decomposed,

$$
m=m_{A}+m_{B},
$$

into the components corresponding to the ferromagnetic slabs $A$ and $B$. We have obtained the following formulae for the coupling (taking $m_{A} \cdot m_{B}=m_{A} m_{B} \cos \vartheta$ ):

$$
J_{0}=-\frac{1}{A} \int \mathrm{d} r\left[\frac{2}{3} \frac{\hbar^{2}}{2 m} \frac{k^{2} \mathrm{~F}(n)}{n}+\frac{4 \varepsilon_{x}(n)}{9 n}+\ldots\right] m_{A} m_{B},
$$




$$
J_{1}=-\frac{1}{A} \int \mathrm{d} r\left[\frac{4}{81} \frac{\hbar^{2}}{2 m} \frac{k_{F}^{2}(n)}{n}+\frac{20 \varepsilon_{x}(n)}{243 n}+\ldots\right]\left(\frac{m_{A} m_{B}}{n}\right)^{2} .
$$

Here, $n$ is the local electron density, $k_{\mathrm{F}}(n)=\left(3 \pi^{2} n\right)^{1 / 3}, \varepsilon_{x}(n)$ - the exchange energy in the local density approximation, $m$ - the electron mass, $A$ - the area of the multilayer and $m_{A}$ and $m_{B}$ are the spin polarization connected with the magnetic slabs $A$ and $B$, respectively. Our formulae for the coupling are given in terms of the electron density and the spin polarization of the multilayer.

The spin densities of the valence electrons at the interface are calculated by .applying a method described in Ref. [6], which was used for calculations of the electron density at a metal surface. The spin density of the valence electrons at the interface is calculated in the Lang-Kohn jellium, where the density of the external charge serves as a parameter. We calculate the spin densities with up and down spins independently. The density of the external charge corresponds to the spin density of the valence electrons for the bulk materials, e.g. calculating the spin density of the up valence electrons, the density of the positive background and the bulk down electron density serve as an effective external charge. The induced polarization at the interface $\mathrm{Fe} / \mathrm{Cu}$ is used for numerical estimation of the coupling. If the spacer does not contain Fe impurities, the main contribution to the coupling comes from the magnetic slabs at the interfaces since the polarization of the nonmagnetic metal is small in comparison with the spontaneous polarization of the magnetic slabs. In the numerical calculation of the coupling, the density of the $\mathrm{Fe} d$-electrons (divided into the core $T_{2 \mathrm{~g}}$ up electrons and the valence electrons) is taken only. The spin polarization of the valence Fe electrons is pointed in the opposite direction with respect to the polarization of the $T_{2 \mathrm{~g}}$ up electrons, since the kinetic energy does not prefer the spin polarization and is greater in comparison with the exchange term of the energy potential. The spin polarization of the bulk Fe valence electrons is obtained by taking equations for the electron density and the spin polarization of the $d$-electrons. The ratio (spontaneous polarization/density of $d$-electrons) weakly depends on the space coordinate and is taken to be constant, in our numerical estimation of the coupling. The bilinear and biquadratic coupling vs. the number of monolayers, $N_{\mathrm{Cu}}$, of the spacer are shown in Figs. 1 and 2.

In this paper, general analytical formulae for the bilinear and biquadratic coupling are given. The electron density and the spin polarization of the magnetic multilayer serve as variables. The mechanism of the coupling is based on the effect of the magnetic configuration on the spin polarization of the magnetic multilayer. The coupling is estimated numerically within a jellium approximation and calculations restricted to the $d$-electrons of magnetic bulk layers. The couplings vs. the number of monolayers of the spacer are shown in Figs. 1 and 2. The calculated value of the amplitude of the bilinear coupling is somewhat greater in comparison with experimental data for ultrathin multilayers, $c$. [7]. The bilinear coupling shows two oscillation periods. The biquadratic coupling strongly decreases with the thickness of the spacer layer and for a big thickness is three orders of magnitude smaller than experimental data [7]. This discrepancy is due to the presence of Fe atoms in the nonmagnetic layer of the real magnetic multilayers. The impurity Fe atoms change the polarization of the spacer layer and strongly intensify the 


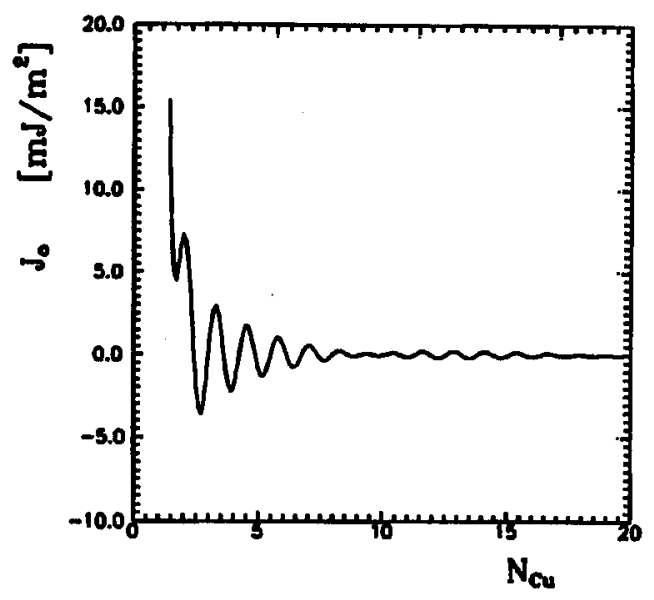

Fig. 1. The oscillatory bilinear coupling, $J_{0}$, vs. the number of monolayers $N_{\mathrm{Cu}}$ of the spacer $\mathrm{Cu}$ film.

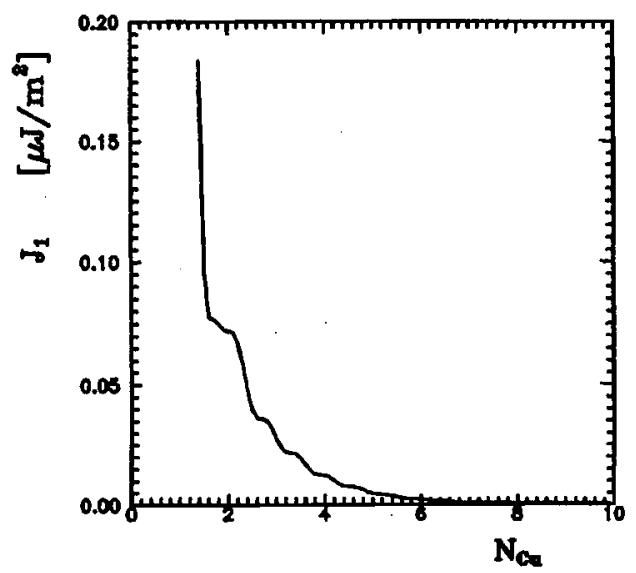

Fig. 2. The biquadratic coupling, $J_{1}$, vs. the number of monolayers $N_{\mathrm{Cu}}$ of the spacer $\mathrm{Cu}$ film.

biquadratic coupling. Admixture of magnetic atoms, in the spacer layer, leads to the high dependence of the coupling on the temperature. Our biquadratic coupling is in a qualitative agreement with the result of Ref. [8]. We hope to improve our numerical results by calculating the electron density in the Hohenberg-Kohn formalism of the density functional theory and the core $T_{2 \mathrm{~g}} d$-electrons solving the Kohn-Sham equation.

I would like to acknowledge some helpful discussion with R. Ferchmin. This work was supported by the Committee for Scientific Research grant No. 2P302 00507. 


\section{References}

[1] M. Rühring, R. Schäfer, A. Hubert, R. Mosler, J.A. Wolf, S. Demokritov, P. Grünberg, Phys. Status Solidi A 125, 653 (1991).

[2] J.C. Slonczewski, Phys. Rev. Lett. 67, 3172 (1991).

[3] D.M. Edwards, J.M. Ward, J. Mathon, J. Magn. Magn. Mater. 126, 380 (1993).

[4] Lie-Ming Li, Fu-Cho Pu, Phys. Rev. B 51, 3640 (1995).

[5] W. Kohn, P. Vashishta, in: Theory of the Inhomogeneous Electron Gas, Eds. S. Lundqvist, N.H. March, Plenum, New York 1983, p. 79.

[6] N.D. Lang, W. Kohn, Phys. Rev. B 7, 3541 (1973).

[7] Heinrich, Z. Celinski, J.F. Cochran, A.S. Arrott, K. Myrtle, S.T. Purcell, Phys. Rev. $B$ 47, 5077 (1993).

[8] J. d'Albuquerque e Castro, M.S. Ferreira, R.B. Muniz, in: Colloquium Digest of 14th Int. Coll. on Magnetic Films and Surfaces, Düsseldorf 1994, Eds. E. Kisker, G. Bayreuther, Heinrich-Heine-Universität, Düsseldorf 1994, Eds. E. Kisker, G. Bayreuther, Heinrich-Heine-Universität, Düsseldorf 1994, p. 553. 